\title{
Short-Scale Turbulent Fluctuations Driven by the Electron-Temperature Gradient in the National Spherical Torus Experiment
}

\author{
E. Mazzucato, ${ }^{1, *}$ D. R. Smith, ${ }^{1}$ R. E. Bell, ${ }^{1}$ S. M. Kaye, ${ }^{1}$ J. C. Hosea, ${ }^{1}$ B. P. LeBlanc, ${ }^{1}$ J. R. Wilson,,${ }^{1}$ P. M. Ryan, ${ }^{2}$ \\ C. W. Domier, ${ }^{3}$ N. C. Luhmann, Jr., ${ }^{3}$ H. Yuh, ${ }^{4}$ W. Lee, ${ }^{5}$ and H. Park ${ }^{5}$ \\ ${ }^{1}$ Princeton Plasma Physics Laboratory, Princeton University, Princeton, New Jersey 08543, USA \\ ${ }^{2}$ Oak Ridge National Laboratory, Oak Ridge, Tennessee 37831, USA \\ ${ }^{3}$ University of California at Davis, Davis, California 95616, USA \\ ${ }^{4}$ Nova Photonics Inc., Princeton, New Jersey 08540, USA \\ ${ }^{5}$ Department of Physics, POSTECH, Pohang 790-784, Korea
}

(Received 13 May 2008; published 11 August 2008)

\begin{abstract}
Measurements with coherent scattering of electromagnetic waves in plasmas of the National Spherical Torus Experiment indicate the existence of turbulent fluctuations in the range of wave numbers $k_{\perp} \rho_{e}=$ $0.1-0.4$, corresponding to a turbulence scale length nearly equal to the collisionless skin depth. Experimental observations and agreement with numerical results from a linear gyrokinetic stability code support the conjecture that the observed turbulence is driven by the electron-temperature gradient.

PACS numbers: 52.55.Fa, 52.35.Qz, 52.35.Ra
\end{abstract}

After more than 50 years of research, the causes of anomalous plasma transport in tokamaks are still an outstanding issue. The plasma physics literature abounds with explanations of this important phenomenon [1-3] — mostly invoking some turbulent process-but none is based on a self-consistent theory together with a comprehensive set of measurements.

Particularly difficult to explain is the transport of electron energy. This is the most worrisome since, in a tokamak reactor, a large fraction of the energy of charged fusion products-necessary to sustain the fusion reactionswould be released directly to the electrons. Various theories and numerical simulations [4-9] support the conjecture that anomalous electron transport may arise from an electron gyroscale turbulence driven by the electrontemperature gradient (ETG) instability. However, even though limited circumstantial evidence has been presented in Ref. [10] on the role of the ETG turbulence in plasma transport on Tore Supra, and measurements on FT-2 of fluctuations with an electron gyroscale have been reported in Ref. [11], the existence of a turbulence driven by the ETG instability has never been proved experimentally in tokamaks.

In this Letter, we present the results of an experimental study of short-scale turbulent fluctuations driven by the electron-temperature gradient in plasmas of the National Spherical Torus Experiment (NSTX). These plasmas are uniquely suited for the study of the physics of electron transport since, while the confinement of ions in NSTX plasmas is very often at or near neoclassical levels, that of electrons is anomalous in all operational regimes [12].

Plasma density fluctuations were measured with coherent scattering of electromagnetic waves, a process that can be characterized by an effective differential cross section per unit volume

$$
\sigma=\left(e / m c^{2}\right)^{2} S(\boldsymbol{k}, \omega)
$$

where $S(\boldsymbol{k}, \omega)$ is the spectral density of fluctuations [13]. The frequency $(\omega)$ and wave vector $(\boldsymbol{k})$ of fluctuations must satisfy the energy and momentum conservation, i.e., $\omega=\omega_{s}-\omega_{i}$ and $\boldsymbol{k}=\boldsymbol{k}_{s}-\boldsymbol{k}_{i}$, where superscripts $s$ and $i$ refer to scattered and incident waves, respectively. Since for the topic of this Letter $\omega_{s} \approx \omega_{i}$ and $k_{s} \approx k_{i}$, the scattering angle $\theta$ must satisfy the Bragg condition

$$
k=2 k_{i} \sin (\theta / 2) \text {. }
$$

The NSTX scattering system employs a probing wave with a frequency of $280 \mathrm{GHz}$, together with a five-channel heterodyne receiver capable of providing full information on the frequency spectrum of measured signals. Its novel scattering geometry (Fig. 1) takes advantage of the large curvature of magnetic field lines and the strong anisotropy of short-scale turbulence in tokamaks-characterized by $k_{\|} \ll k_{\perp}$ (with $k_{\|}$and $k_{\perp}$ the wave vector components parallel and perpendicular to the magnetic field, respectively) - to attain a radial resolution of $\pm 2.5 \mathrm{~cm}$ together with a wave number resolution of $\pm 1 \mathrm{~cm}^{-1}[14,15]$. Probe and scattered waves lie nearly on the equatorial midplane such that the wave vectors of detected fluctuations (in the range 5-20 $\mathrm{cm}^{-1}$ ) are mainly perpendicular to the magnetic surfaces. However, they have also small components in both the diamagnetic and the toroidal directions, from which one can infer the phase velocity of fluctuations in the poloidal plane. Finally, steerable optics can position the scattering region from the magnetic axis to the plasma outboard edge.

The experimental results presented in this Letter were obtained in helium plasmas with high harmonic fast wave (HHFW) heating [16]. Use of this radio frequency (rf) technique-where an electromagnetic wave with the frequency $(30 \mathrm{MHz})$ of an ion cyclotron harmonic $(\sim 10)$ is launched into NSTX, where it is absorbed by the electrons-was motivated by its ability to produce electron- 

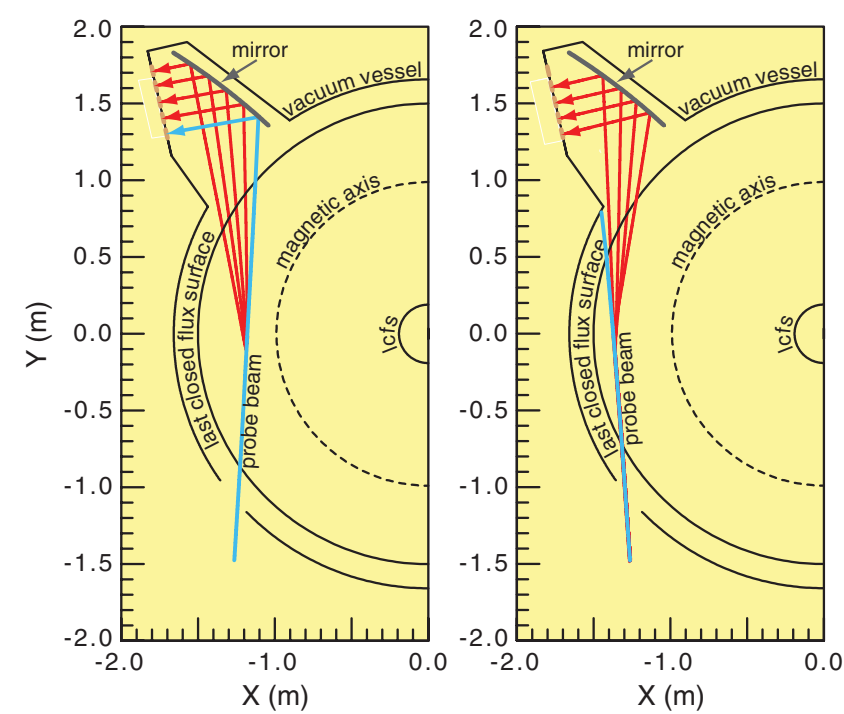

FIG. 1 (color). Probe beam (blue line) and scattered waves (red line) for detection of core (left) and edge (right) fluctuations.

temperature $\left(T_{e}\right)$ profiles with large central values and steep gradients. An example is in Fig. 2, showing the electron-temperature and density profiles during HHFW heating $(0.15-0.40 \mathrm{~s})$ with $1.2 \mathrm{MW}$ of $\mathrm{rf}$ power in a plasma with a minor radius of $0.65 \mathrm{~m}$, a major radius of $0.85 \mathrm{~m}$, an elongation of 2 , a magnetic field of $0.55 \mathrm{~T}$, and a current of $700 \mathrm{kA}$. The latter values of magnetic field and plasma current were chosen to minimize the spurious effects of MHD turbulence. Because of the low plasma density, i.e., a weak electron-ion coupling, the ion temperature $\left(T_{i}\right)$ re-

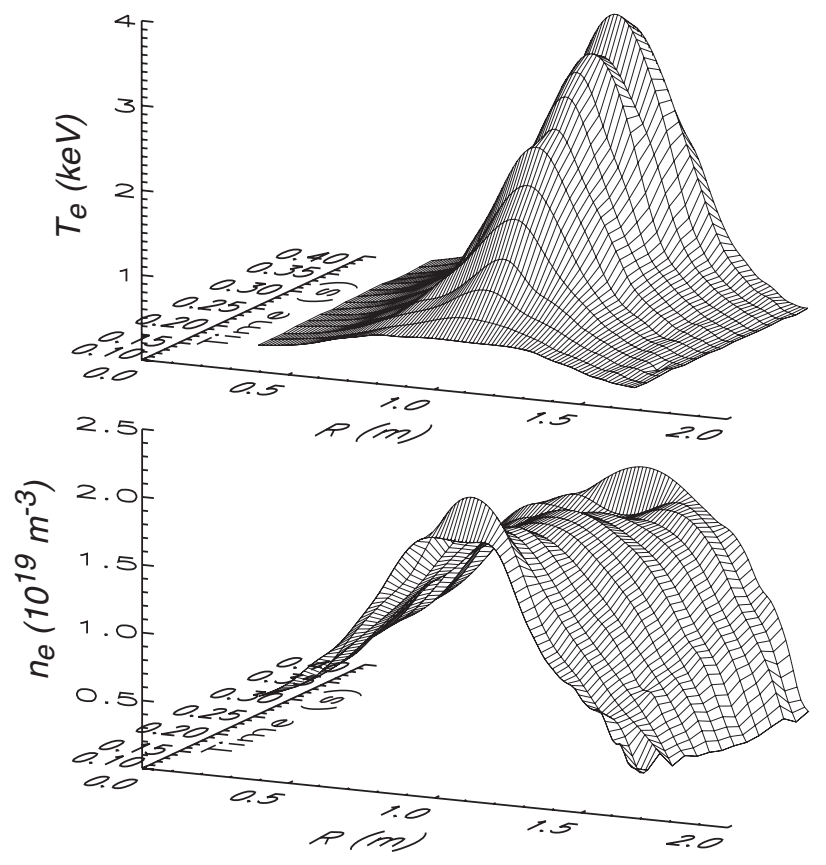

FIG. 2. Electron-temperature (top) and density (bottom) profiles with 1.2 MW of HHFW heating (0.15-0.40 s).

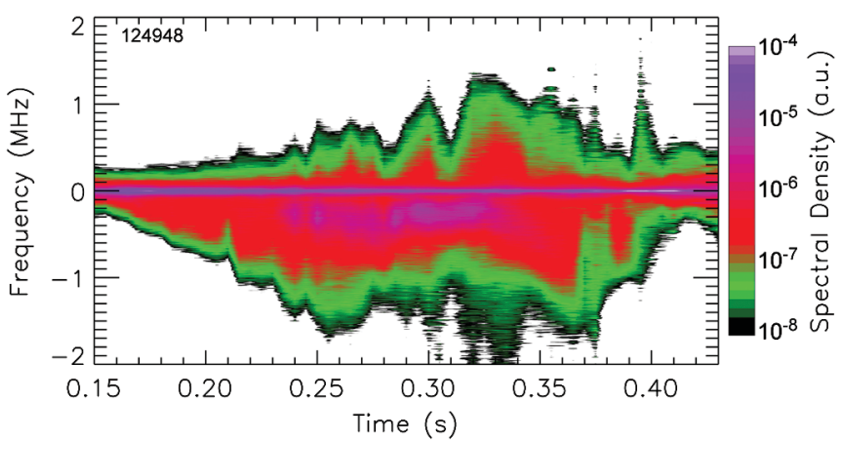

FIG. 3 (color). Logarithmic contour plot of spectral density of fluctuations with $k_{\perp} \rho_{e}=0.2-0.4$ at $R=1.2 \mathrm{~m}$. Negative frequencies correspond to wave propagation in the electron diamagnetic direction.

mained nearly constant (with central values of $0.7-$ $1.0 \mathrm{keV})$.

Figure 3 shows the time evolution of the power density of measured fluctuations with a wave number of $k_{\perp}=$ $14 \mathrm{~cm}^{-1}$ at $R=1.2 \mathrm{~m}$, corresponding to a range of $k_{\perp} \rho_{e}=0.2-0.4$ (with $\rho_{e}$ the electron gyroradius), $k_{\perp} \rho_{s}=$ 8.5-17 (with $\rho_{s}$ the ion gyroradius at the electron temperature), and $k_{\perp} \rho_{i}=8-10$ (with $\rho_{i}$ the ion gyroradius). Such a scale implies that the source of observed fluctuations is not the ion-temperature gradient (ITG) mode, which is characterized by $k_{\perp} \rho_{i}<1$ [1-3]. This mode is also excluded by the frequency asymmetry of measured spectra (Fig. 3), indicating that fluctuations propagate in the electron diamagnetic direction.

It is interesting to note that, for the plasma density in Fig. $2, k_{\perp} \delta_{s k} \sim 2$ (with $\delta_{s k}=c / \omega_{p e}$ the collisionless skin depth). This is not surprising since for sufficiently high electron betas, such as those in the present experiment $(3 \%-6 \%)$, the characteristic turbulence scale length is expected to be of the order of the electron skin depth $[4,17]$.

The observed fluctuations appear to be related to the electron-temperature gradient, as illustrated in Fig. 4, where the total spectral power of fluctuations of Fig. 3 is compared with the scale of $T_{e}$ at the radius of measurements [defined as $L_{T_{e}}=\left(d \ln T_{e} / d r\right)^{-1}$ ]. Note that plasma fluctuations begin to rise at the beginning of the rf pulse,

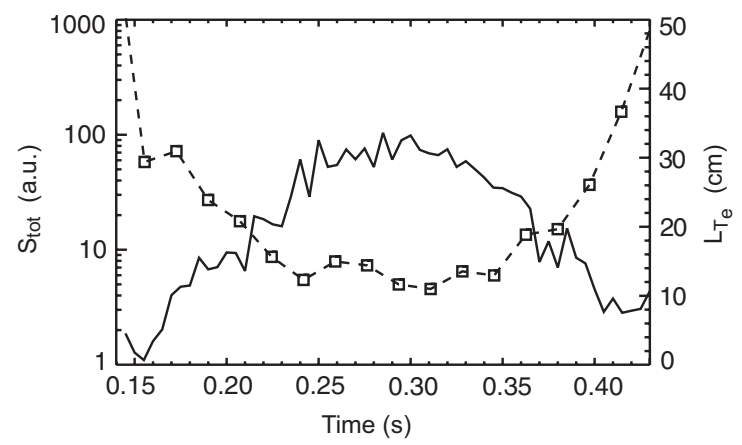

FIG. 4. Frequency integrated spectral density $S_{\text {tot }}$ (solid line) and radial scale $L_{T_{e}}$ (dashed line) for the case of Fig. 3. 

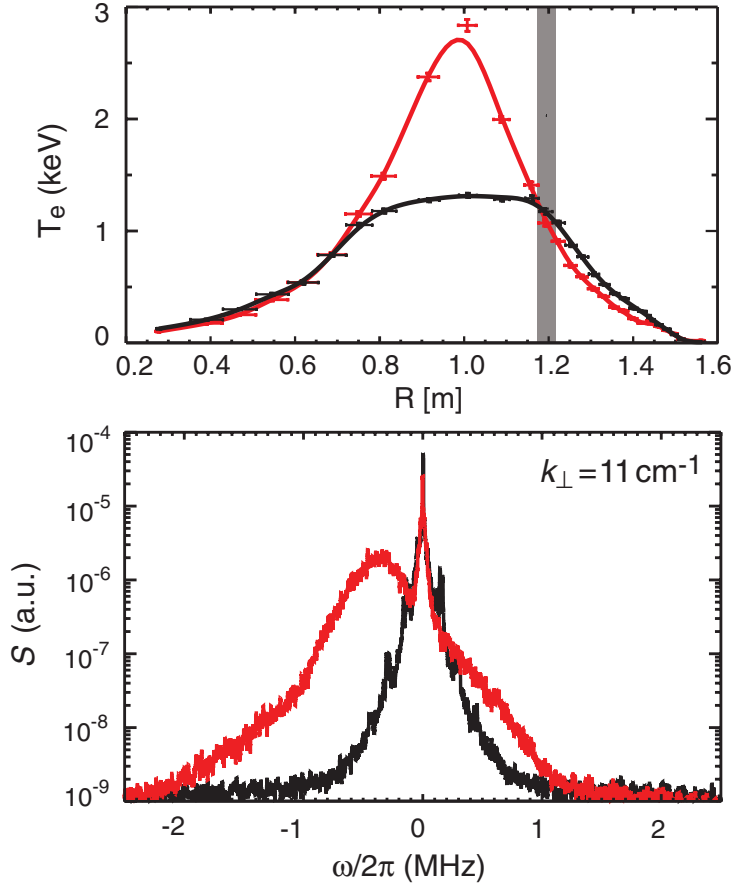

FIG. 5 (color). Temperature profiles (top) and spectral density of fluctuations (bottom) at 0.3 (red line) and $0.43 \mathrm{~s}$ (black line). The shaded area (top) is the measurement location where $L_{T_{e}}$ is 15 and $50 \mathrm{~cm}$, respectively. Negative frequencies (bottom) correspond to wave propagation in the electron diamagnetic direction.

when the value of $L_{T_{e}}$ begins to drop, and decrease towards the end of the pulse, when the opposite occurs.

The same phenomenon is illustrated in Fig. 5, showing the electron-temperature profile and the spectrum of fluctuations at $0.3 \mathrm{~s}-$ when fluctuations are near their maximum - and at $0.43 \mathrm{~s}-30 \mathrm{~ms}$ after the end of the rf pulse when the electron-temperature profile has collapsed and flattened on a wide central region. While both spectra contain a central narrow symmetric feature, which is caused by a spurious stray radiation, the spectrum at $0.3 \mathrm{~s}$ displays a strong Doppler-shifted component, which is the signal of coherent wave scattering from plasma fluctuations propagating in the electron diamagnetic direction. A difference of a factor of 3 in $L_{T_{e}}$ at the two times of measurement clearly demonstrates the dependence of plasma turbulence on the radial scale of $T_{e}$.

Short-scale turbulent fluctuations were detected on the outer region of the plasma column $(R=1.35 \mathrm{~m})$ as well. Some results are displayed in Fig. 6, showing the spectral density of fluctuations with wave numbers in the range $k_{\perp} \rho_{e}=0.1-0.2$. Again, the turbulence scale length is of the order of the collisionless skin depth $\left(k_{\perp} \delta_{s k}=1-2\right)$. Also, as in the case of core fluctuations, wave numbers are outside the range of the ITG mode, and their propagation is in the electron diamagnetic direction (corresponding to positive frequencies for the scattering configuration of these measurements).
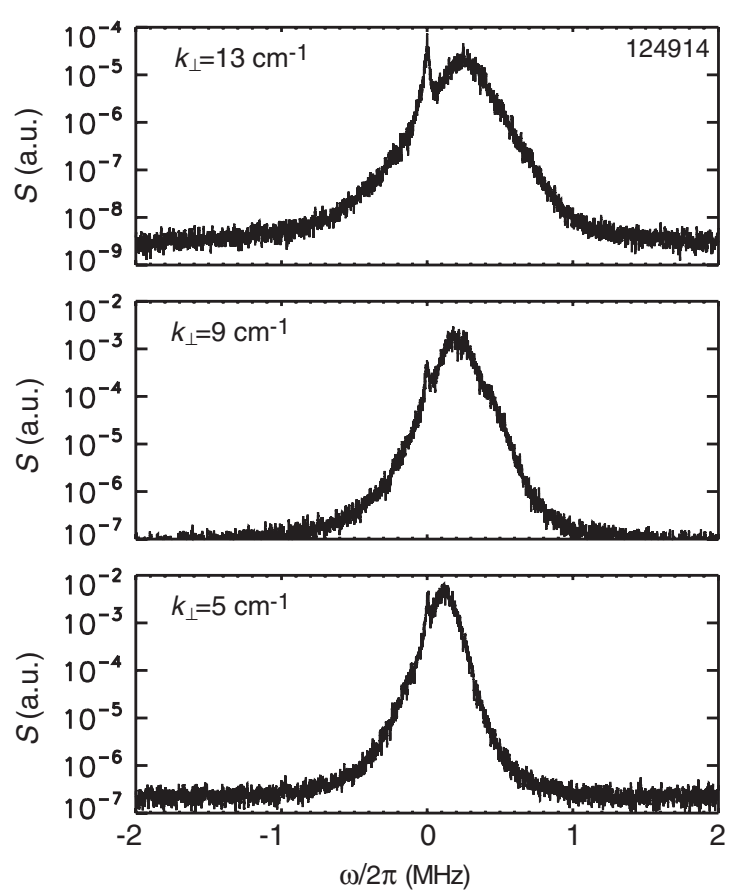

FIG. 6. Spectral density of fluctuations in the range of wave numbers $k_{\perp} \rho_{e}=0.1-0.2$ at $R=1.35 \mathrm{~m}$. Positive frequencies correspond to wave propagation in the electron diamagnetic direction.

In an attempt to determine the source of observed fluctuations, we employed a linear version of the GS2 stability code [18] to obtain the normalized critical gradient $\left(R / L_{T_{e}}\right)_{\text {crit }}$ for the onset of the ETG instability. This code solves the gyrokinetic Vlasov-Maxwell equations including both passing and trapped particles and electromagnetic effects, as well as a Lorentz collision operator. The results are shown in Fig. 7, where the critical gradient is compared with the measured normalized temperature gradient $R / L_{T_{e}}$ for the case of Fig. 3. From this, we conclude that the ETG mode is indeed unstable over most of the rf pulse where the

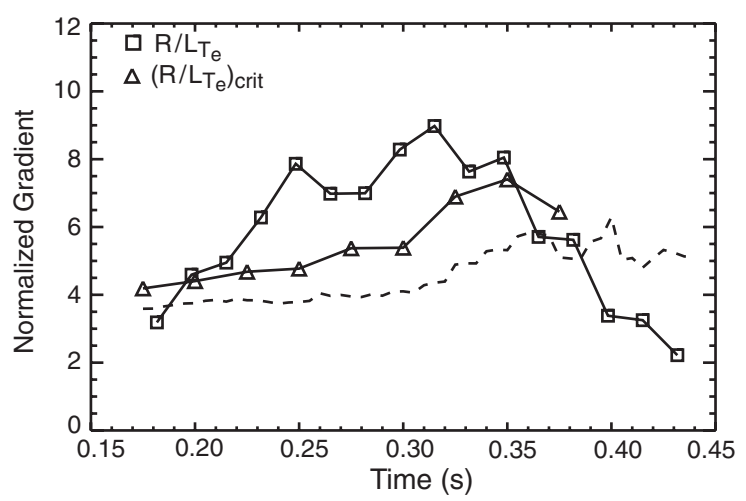

FIG. 7. Time evolution of measured gradient $R / L_{T_{e}}$ (squares) and GS2 critical gradient $\left(R / L_{T_{e}}\right)_{\text {crit }}$ for the onset of the ETG mode (triangles). The dashed line is the critical gradient from Ref. [19]. 


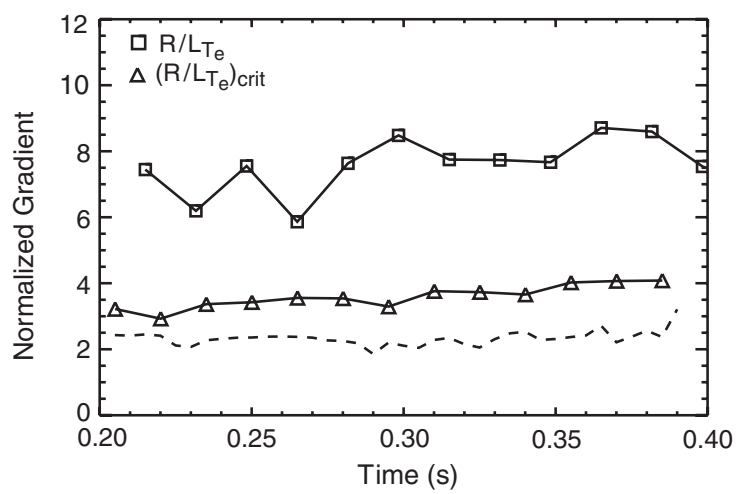

FIG. 8. Same as in Fig. 7 for the case of Fig. 6.

critical gradient is smaller than the electron-temperature gradient. Comparison with Fig. 4 shows that the level of measured fluctuations correlates with the departure of the temperature gradient from the critical gradient.

Figure 7 displays also an empirical value of the normalized critical gradient that was derived in Ref. [19] with a best fit of numerical results from the linear GS2 code using a set of model tokamak configurations. It is given by

$$
\left(R / L_{T_{e}}\right)_{\text {crit }}=\left(1+Z_{\text {eff }} T_{e} / T_{i}\right)(1.3+1.9 s / q)(1-1.5 \varepsilon),
$$

where $Z_{\text {eff }}$ is the ionic effective charge $(\sim 2.5$ in the present experiment), $q$ is the magnetic safety factor, $s=(r / q) \times$ $(d q / d r)$ is the magnetic shear, and $\varepsilon=r / R$ is the inverse aspect ratio. This algebraic expression-showing the stabilizing role of the temperature ratio $T_{e} / T_{i}$ and the magnetic shear-gives values of critical gradient that are not very different from those that we obtained from the linear GS2 code using the exact plasma configuration of our experiment.

Similar plots are displayed in Fig. 8 for the case of outboard fluctuations (Fig. 6), showing again that fluctuations coincide with a temperature gradient that is larger

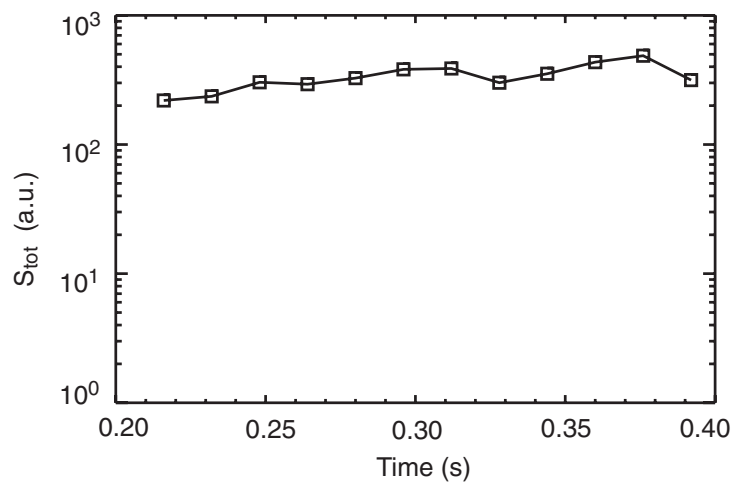

FIG. 9. Frequency integrated spectral density $S_{\text {tot }}$ for fluctuations with $k_{\perp}=13$ of Fig. 6. than the critical gradient. At this plasma location, however, plasma conditions are not affected significantly by the rf heating; i.e., the temperature and critical gradients remain nearly constant, and consequently the level of fluctuations remains nearly constant as well (Fig. 9).

In conclusion, turbulent density fluctuations have been observed in NSTX plasmas in the range of wave numbers $k_{\perp} \rho_{e}=0.1-0.4$, corresponding to a turbulence scale length of the order of the collisionless skin depth. Large values of $k_{\perp} \rho_{i}$ and a strong correlation with $R / L_{T_{e}}$ seem to exclude the ITG mode as the source of turbulence. Experimental observations and an agreement with numerical results from the linear gyrokinetic GS2 code support the conjecture that the observed turbulence is driven by the electron-temperature gradient. Additional experiments together with nonlinear numerical simulations of plasma turbulence are needed to assess the role of observed fluctuations on plasma transport.

This work was supported by U.S. Department of Energy Contract No. DE-AC02-76CH03073 and Grant No. DEFG-02-99ER54518.

\section{*mazzucato@pppl.gov}

[1] B. Coppi and G. Rewoldt, in Advances in Plasma Physics, edited by A. Simon and W. B. Thompson (Wiley, New York, 1976), Vol. 6, p. 421.

[2] W. Horton, Rev. Mod. Phys. 71, 735 (1999).

[3] J. W. Connor and H. R. Wilson, Plasma Phys. Controlled Fusion 36, 719 (1994).

[4] W. Horton et al., Phys. Plasmas 7, 1494 (2000).

[5] W. Dorland, F. Jenko, M. Kotschenreuther, and B. N. Rotgers, Phys. Rev. Lett. 85, 5579 (2000).

[6] F. Jenko and W. Dorland, Phys. Rev. Lett. 89, 225001 (2002).

[7] W. M. Nevins et al., Phys. Plasmas 13, 122306 (2006).

[8] A. M. Dimits et al., Nucl. Fusion 47, 817 (2007).

[9] R.E. Waltz, J. Candy, and M. Fahey, Phys. Plasmas 14, 056116 (2007).

[10] W. Horton, G. T. Hoang, C. Bourdelle, X. Garbet, M. Ottaviani, and L. Colas, Phys. Plasmas 11, 2600 (2004).

[11] A. D. Gurchenko et al., Nucl. Fusion 47, 245 (2007).

[12] S. M. Kaye et al., Phys. Rev. Lett. 98, 175002 (2007).

[13] M.N. Rosenbluth and N. Rostoker, Phys. Fluids 5, 776 (1962).

[14] E. Mazzucato, Phys. Plasmas 10, 753 (2003).

[15] E. Mazzucato, Plasma Phys. Controlled Fusion 48, 1749 (2006).

[16] J. Hosea et al., Phys. Plasmas 15, 056104 (2008).

[17] B. Coppi, in Collective Phenomena in Macroscopic Systems, edited by G. Bertin (World Scientific, Singapore, 2007).

[18] M. Kotschenreuther, G. Rewoldt, and W. M. Tang, Comput. Phys. Commun. 88, 128 (1995).

[19] F. Jenko, W. Dorland, and G. W. Hammett, Phys. Plasmas 8, 4096 (2001). 